\title{
CORRECTION
}

\section{Author Correction: Single-cell RNA-seq highlights intra-tumoral heterogeneity and malignant progression in pancreatic ductal adenocarcinoma}

Junya Peng ${ }^{1}$, Bao-Fa Sun ${ }^{2,3,4}$, Chuan-Yuan Chen ${ }^{2,3}$, Jia-Yi Zhou ${ }^{2,3}$, Yu-Sheng Chen iD $^{2,3}$, Hao Chen ${ }^{5}$, Lulu Liu ${ }^{1}$, Dan Huang ${ }^{1}$, Jialin Jiang ${ }^{5}$, Guan-Shen Cui ${ }^{2,3}$, Ying Yang ${ }^{2,3,4}$, Wenze Wang ${ }^{6}$, Dan Guo ${ }^{1,7}$, Menghua Dai ${ }^{5}$, Junchao Guo ${ }^{5}$, Taiping Zhang ${ }^{5}$, Quan Liao ${ }^{5}$, Yi Liu ${ }^{8}$, Yong-Liang Zhao ${ }^{2,3,4}$, Da-Li Han ${ }^{2,3,4}$, Yupei Zhao ${ }^{5,8}$, Yun-Gui Yang $\mathbb{i D}^{2,3,4}$ and Wenming $\mathrm{Wu}^{5}$

Cell Research (2019) 29:777; https://doi.org/10.1038/s41422-019-0212-1

Correction to: Cell Research (2019) https://doi.org/10.1038/ s41422-019-0195-y, published online 4 July 2019
In the initial published version of this Article, there was an error in author information. Da-Li Han (handl@big.ac.cn) is a co-first author and senior author. This correction does not affect the description of the results or the conclusions of this work.

\footnotetext{
${ }^{1}$ Department of Medical Research Center, Peking Union Medical College Hospital, Chinese Academy of Medical Science \& Peking Union Medical College, Beijing 100730, China; ${ }^{2}$ CAS Key Laboratory of Genomic and Precision Medicine, Collaborative Innovation Center of Genetics and Development, College of Future Technology, Beijing Institute of Genomics, Chinese Academy of Sciences, Beijing 100101, China; ${ }^{3}$ University of Chinese Academy of Sciences, Beijing 100049 , China; ${ }^{4}$ Institute of Stem Cell and Regeneration, Chinese Academy of Sciences, Beijing 100101, China; ${ }^{5}$ Department of General Surgery, Peking Union Medical College Hospital, Chinese Academy of Medical Science \& Peking Union Medical College, Beijing 100730, China; ${ }^{6}$ Department of Pathology, Peking Union Medical College Hospital, Chinese Academy of Medical Science \& Peking Union Medical

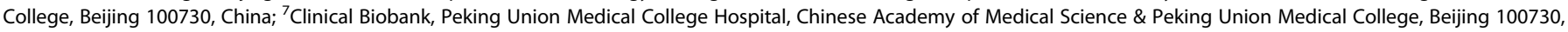
China and ${ }^{8}$ Tsinghua University-Peking University Joint Center for Life Sciences, School of Medicine, Tsinghua University, Beijing 100084, China

Correspondence: Yupei Zhao (zhao8028@263.net) or Yun-Gui Yang (ygyang@big.ac.cn) or Wenming Wu (doctorwuu@126.com)

These authors contributed equally: Junya Peng, Bao-Fa Sun, Chuan-Yuan Chen, Jia-Yi Zhou, Yu-Sheng Chen, Da-Li Han
}

Published online: 13 August 2019 